\title{
Experiencia en el tratamento del dolor crónico con estimulación transcraneal en pacientes del ISSSTE
}

\author{
Guadalupe Y. Medina-Chamu*
}

Fisioterapia, Hospital de Alta Especialidad Centenario de la Revolución Mexicana, Instituto de Seguridad y Servicios Sociales de los Trabajadores del Estado (ISSSTE), Cuernavaca, Morelos, México

\begin{abstract}
Resumen
El objetivo de este estudio es conocer la experiencia en el tratamiento del dolor crónico con estimulación transcraneal en pacientes del Hospital de Alta Especialidad Centenario de la Revolución Mexicana, del Instituto de Seguridad y Servicios Sociales de los Trabajadores del Estado (ISSSTE). Se aplicó un diseño descriptivo (observacional), en el cual participaron 40 pacientes con dolor crónico que acudieron al Servicio de Rehabilitación del ISSSTE Centenario de la Revolución Mexicana, quienes iniciaron un tratamiento de 10 sesiones de terapia. Se utilizó como material entrevistas y expedientes clínicos para plasmar los resultados, mismos resultados que evidencian que existe una experiencia favorable, buena y con un gran resultado de mejoría. Por lo tanto, se concluye que este artículo proporcionará datos relevantes para conocer la experiencia y con ello la efectividad de la estimulación transcraneal con corriente directa en el dolor crónico. De la muestra estudiada, todos los pacientes tuvieron una experiencia satisfactoria, con una mejoría por arriba del 50\% en disminución del dolor. La estimulación transcraneal está siendo investigada mucho en los últimos años, pero aquí en nuestro medio aún es muy desconocida.
\end{abstract}

Palabras clave: Dolor crónico. Estimulación transcraneal. Corriente directa.

\section{Experience in the treatment of chronic pain with transcranial stimulation in ISSSTE patients}

\begin{abstract}
The objective of this study is to know the experience in the treatment of chronic pain with transcranial stimulation in patients of the High Specialty Hospital centennial of the Mexican Revolution, a descriptive design was applied (observational) which involved 40 patients with chronic pain that came to the rehabilitation of ISSSTE centennial of the revolution, who started a treatment of 10 therapy sessions. It was used as material interviews and clinical records for translating the results, same results that show that there is a favorable experience, good and with a great result of improvement. It is therefore concluded that this study will provide data relevant to the experience and the effectiveness of the transcranial stimulation with direct current in chronic pain. Of the sample studied, all patients had a satisfactory experience, taking an improvement above 50\% in decrease of pain. Transcranial stimulation is being investigated a lot in the last few years but here in our midst is still unknown.
\end{abstract}

Key words: Chronic pain. Transcranial stimulation. Direct current.

\footnotetext{
Correspondencia:

Fecha de recepción: 17-08-2020

*Guadalupe Y. Medina-Chamu

E-mail: yarelyl_0189@hotmail.com

DOI: 10.24875/REMQ.20000027

Disponible en internet: 04-06-2021

Rev Esp Méd Quir. 2020;25:82-7

www.remq-issste.com

1665-7330 / @ 2021 Revista de Especialidades Médico-Quirúrgicas. Publicado por Permanyer México SA de CV. Este es un artículo Open Access bajo la licencia CC BY-NC-ND (http://creativecommons.org/licenses/by-nc-nd/4.0/).
} 


\section{Introducción}

Según la Asociación Internacional para el Estudio del Dolor (IASP, por sus siglas en inglés), el dolor es «una experiencia sensorial y emocional desagradable asociada con el daño tisular real o potencial, o se describe en términos de dicho daño"'. Asimismo, dicha asociación clasifica el dolor en agudo y crónico, este último está relacionado con diferentes enfermedades o condiciones clínicas.

La Organización Mundial de la Salud reconoce el dolor como un importante problema de salud pública mundial. En el 2017 identificó la necesidad de un tratamiento mejorado y estandarizado. Su diagnóstico plantea un reto y su tratamiento se complica cuando tiene una causa multifactorial. La prevalencia del dolor crónico varía de un 16 a un $70 \%$, es más frecuente en mujeres que en hombres y se asocia a enfermedades degenerativas y a edad superior de 65 años $^{2}$.

La estimulación transcraneal con corriente directa es una forma de estimulación eléctrica que consiste en la aplicación de dos electrodos de superficie (ánodo y cátodo) colocados por encima del cuero cabelludo, que administran una corriente eléctrica directa de baja amplitud (0.5-2 mA) capaz de atravesar el hueso craneal y modular el área cerebral seleccionada. Dicha corriente induce cambios en el potencial de reposo de la membrana neuronal.

El dolor crónico se ha reconocido como un dolor que persiste más allá del tiempo normal de curación ${ }^{3}$ y, por lo tanto, carece de la función de advertencia aguda de la nocicepción fisiológica ${ }^{4}$.

Se considera dolor crónico cuando este persiste o recurre durante más de 3 a 6 meses $^{5}$. Representa una condición frecuente que afecta a aproximadamente el $20 \%$ de las personas en el mundo y que generan del 15 al $20 \%$ de consultas médicas ${ }^{6}$. Por tanto, esta condición debe recibir mayor atención como una prioridad de atención en los servicios de salud a nivel global, debido a que el alivio del dolor se considera como un derecho humano?.

El dolor crónico es un problema multifactorial que incluye componentes tanto físicos como psicológicos, como ansiedad, movilidad reducida, alteraciones del sueño y del apetito y depresión ${ }^{8}$. Estos síntomas están relacionados con una reducción demostrable de la calidad de vida de los pacientes y una limitación de su desempeño laboral y de la función social ${ }^{9}$, y requieren al uso frecuente de recursos sanitarios, todo lo que contribuye a generar una carga socioeconómica importante ${ }^{10}$.

Además de su alta frecuencia y su impacto individual y social relevante, el dolor crónico ha demostrado ser un importante contribuyente al aumento de la utilización de la atención médica, a la reducción de la productividad laboral y, en consecuencia, a los grandes costos directos e indirectos.

\section{Fisiopatología del dolor}

El dolor es el síntoma mas frecuente, molesto e inquietante en la medicina. Son pocas las enfermedades que no tienen una fase dolorosa y en casi todas el dolor es una característica sin la cual el diagnóstico no se confirma. Por la enorme frecuencia con que surge el dolor, sus características estructurales y fisiológicas asumen importancia especial.

\section{Receptores del dolor y vías aferentes periféricas}

Los receptores del dolor están distribuidos en todo el organismo, en sus estructuras integumentarias y profundas que incluyen las vísceras. Se han identificado dos tipos de fibras aferentes: las fibras $C$ amielínicas muy finas (0.4 a $1.1 \mathrm{~m}$ de diámetro) y las A-delta mielínicas finas ( 1 a $5 \mu \mathrm{m}$ de diámetro).

Es importante reconocer que no todos los dolores son iguales, de tal manera que tendríamos que distinguir muy claro el síndrome doloroso de cada paciente. El síndrome doloroso somático suele referirse como opresivo o punzante, estar bien localizado y está relacionado con daño a estructuras somáticas, como hueso, músculo y tendón. Se transmite primordialmente por fibras A-delta. El síndrome doloroso visceral suele ser más de tipo cólico o sordo, mal definido en su localización y transmitido por fibras amielínicas tipo $\mathrm{C}^{11}$.

\section{Fisioterapia}

\section{Corriente directa transcraneal}

La corriente directa transcraneal (CDT o TDCS) es una técnica con pocos efectos adversos y consiste en la administración de una corriente eléctrica de muy baja intensidad sobre el cuero cabelludo mediante dos electrodos. Ambos electrodos interactúan para crear un campo eléctrico que altera el potencial de 
membrana en reposo y la tasa de descarga espontánea de las células ${ }^{12}$, siendo capaz de influir sobre la actividad cortical del cerebro ${ }^{13,14}$.

La eficacia de la CDT ya ha sido demostrada en ciertas patologías, como es el caso de la depresión, la fibromialgia y la ansiedad. También ha evidenciado efectos beneficiosos en el tratamiento del dolor crónico de origen neuropático en lesionados medulare $\mathrm{s}^{15} \mathrm{O}$ en el tratamiento de la extremidad superior afectada después de un accidente cerebrovascular ${ }^{16}$.

La CDT es capaz de producir fluctuaciones en la actividad neuronal del cerebro importantes para la percepción ${ }^{17,18}$ y la cognición $n^{19,20}$ y, en ultima instancia, influir sobre el aprendizaje motor. Actualmente está surgiendo una nueva corriente de investigación cuyo foco de estudio es la combinación de la CDT junto con otros técnicas para así potenciar su efecto, como en el caso de la realidad virtual ${ }^{21}$. Aunque es una técnica que lleva utilizándose muchos años, su aplicación en fisioterapia es relativamente nueva y requiere más investigación que respalde su efecto y, además, abarque aspectos como el registro y consenso de los parámetros de aplicación.

\section{Técnica de estimulación no invasiva}

\section{La CDT:}

Utiliza electrodos (el ánodo excita y el cátodo inhibe la excitabilidad neuronal).

Aplica una corriente eléctrica galvánica.

Más económico.

Más portabilidad y, por lo tanto, más posibilidad de combinación simultánea.

Más sencilla.

La CDT aplica una corriente eléctrica galvánica para crear un campo eléctrico que altera los potenciales de membrana en reposo y la tasa de descarga espontánea de las células.

\section{Justificación}

Por medio de este estudio se dará a conocer la experiencia de los pacientes con dolor crónico que han sido tratados con estimulación eléctrica transcraneal con corriente directa en el Hospital de Alta Especialidad Centenario de la Revolución Mexicana del ISSSTE, durante el periodo de agosto de 2017 a julio de 2018, mediante la revisión de expedientes clínicos y entrevista en la que se les aplicará la escala visual analógica (EVA), o escala numérica, del dolor.
El tratamiento del dolor crónico con CDT es poco conocida y empleada en nuestro medio, siendo un recurso de gran utilidad para la mejoría de dicha condición de acuerdo con múltiples investigaciones sobre esta modalidad terapéutica.

«El dolor es una experiencia sensorial o emocional desagradable asociada a un daño real o potencial en un tejido, o descrito en términos de dicho daño». No obstante, en ocasiones también se puede experimentar dolor en ausencia de un daño tisular, e incluso, su intensidad puede no ser proporcional con el daño original.

La EVA, introducida por Downie en 1978, es una de las más comúnmente empleadas. El paciente debe asignar al dolor un valor numérico entre dos puntos extremos (0 a 10). Con este tipo de escala el dolor se considera un concepto unidimensional simple y se mide solo según su intensidad. Es útil como instrumento de medida para valorar la respuesta a un tratamiento seleccionado.

Tanto la prevalencia como la intensidad son mayores en las mujeres que en los hombres, aumentando paralelamente a la edad. La relación del dolor con el trabajo muestra valores en general superiores en los hombres que realizan trabajos no manuales y en las mujeres con trabajo manuales.

\section{Objetivos}

- General: conocer la experiencia de los pacientes con dolor crónico que recibieron tratamiento con estimulación transcraneal con corriente directa en el Hospital de Alta Especialidad Centenario de la Revolución Mexicana del ISSSTE.

- Específicos:

- Valorar la disminución del dolor crónico mediante la escala de EVA.

- Conocer la capacidad en las actividades de la vida diaria (AVD) con la disminución del dolor.

\section{Metodología}

\section{Sujetos, material y métodos}

- Sujetos: pacientes con dolor crónico que recibieron tratamiento de estimulación transcraneal con corriente directa.

- Material: entrevista (hojas, pluma, expediente clínico). 
- Método:

- Solicitar los expedientes clínicos que recibieron tratamiento de estimulación transcraneal con corriente directa durante el periodo de agosto de 2017 a julio de 2018.

- Aplicar la entrevista a los pacientes que cumplen criterios de selección.

- Cuantificar la percepción de la disminución del dolor mediante la escala de EVA.

El estudio se llevó a cabo en el Hospital de Alta Especialidad Centenario de la Revolución Mexicana del ISSSTE, en el área de rehabilitación, durante el periodo: agosto de 2017 - julio de 2018.

Se decidió realizar este estudio para valorar la experiencia con la terapia de estimulación transcraneal, ya que estudios recientes reportan que puede ser una alternativa prometedora en la disminución del dolor crónico.

\section{Diseño}

Es un estudio descriptivo (observacional), retrospectivo, transversal, observacional, prolectivo transversal y descriptivo.

\section{Grupos de estudio}

Se estudió a los pacientes con dolor crónico que acudieron al servicio de rehabilitación del ISSSTE Centenario y que recibieron tratamiento de estimulación transcraneal con corriente directa.

\section{Criterios de selección}

- Pacientes con diagnóstico de dolor crónico.

- Pacientes con dolor crónico de ambos sexos.

- Pacientes con dolor crónico mayores de 18 años que recibieron estimulación transcraneal con corriente directa.

- Pacientes con dolor crónico hemodinámicamente estables.

\section{Criterios de exclusión}

- Pacientes con dolor crónico secundario a una enfermedad específica.

- Radiculopatías severas.

- Cáncer.

- Enfermedades psiquiátricas.

\section{Criterios de eliminación}

- Pacientes con dolor crónico que no terminaron el bloque de 10 sesiones de tratamiento.

Muestra no probabilística, se captaron los pacientes que cumplieron todos los criterios de inclusión.

\section{Resultados}

La muestra estudiada fue de 40 pacientes que recibieron estimulación transcraneal con corriente directa que presentaban dolor crónico, de los cuales 30 fueron mujeres (90\%) y 10 hombres (10\%).

Entre los pacientes se encontró que 25 pacientes eran mayores de 50 años y 15 menores de 49 años, con una moda de 45 años.

De acuerdo con la escala de EVA se encontraron 21 pacientes con una mejoría del 10, 14 pacientes con una mejoría del 9, 4 pacientes con una mejoría del 8 y 1 paciente con una mejoría del 7 .

De la población estudiada se determinó que todos nuestros pacientes tuvieron una experiencia satisfactoria en el tratamiento, todos tuvieron una mejoría arriba del $70 \%$.

Por último, se revisó el expediente de cada paciente para conocer la prescripción de ejercicio terapéutico de cada paciente y se encontró en el $100 \%$ de los casos una prescripción del ejercicio terapéutico (resistencia, fuerza y estiramientos) (Figs. 1-4).

\section{Discusión}

De acuerdo con los resultados obtenidos en este trabajo se demostró que el objetivo principal de este estudio se cumplió, al conocer la experiencia de los pacientes con dolor crónico que recibieron tratamiento con estimulación transcraneal con corriente directa en el Hospital de Alta Especialidad Centenario de la Revolución Mexicana del ISSSTE.

Está claro lo que implica 10 sesiones de estimulación transcraneal en el tratamiento del dolor crónico en cuanto a la eficacia de este tratamiento. Cabe resaltar que las 10 sesiones de terapia tienen que cumplirse, ya que son necesarias para poder producir los resultados clínicamente significativos.

Hasta el momento no hay estudios que hayan determinado lo anterior, como antecedentes se ha investigado los agentes físicos para el dolor crónico y los fármacos. 


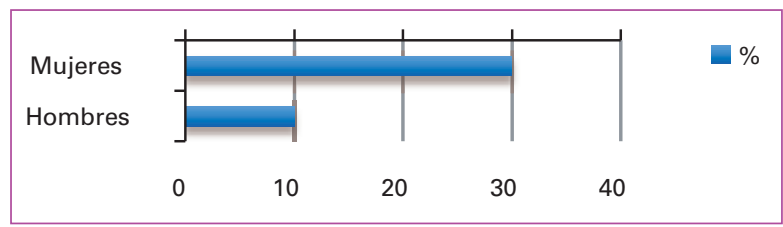

Figura 1. Distribución por sexo.

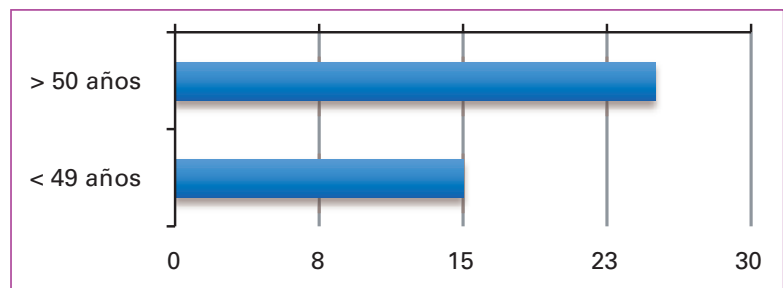

Figura 2. Distribución por edad.

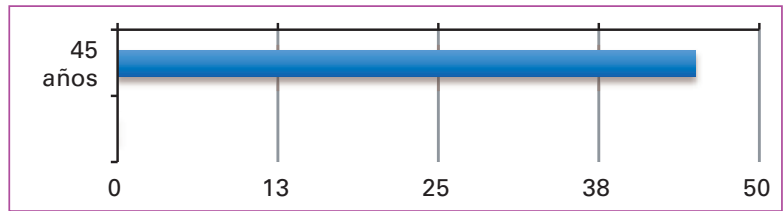

Figura 3. Moda de edad (45 años).

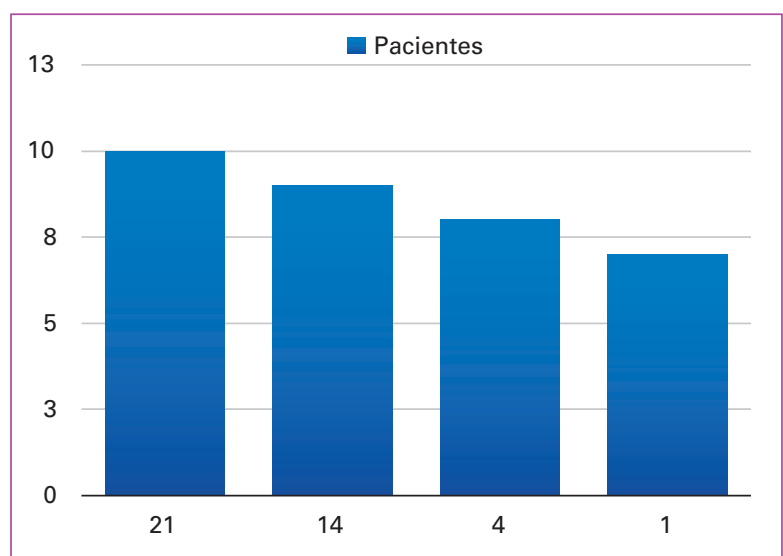

Figura 4. Mejoría según la Escala Visual Analógica

A pesar de ser una técnica poco conocida y empleada, mostró ser eficaz y mejorar el alivio del dolor crónico, ayudando a los pacientes a incorporarse nuevamente a sus actividades de la vida diaria que antes tenían limitación.

Todos nuestros pacientes tuvieron una mejoría de más del $50 \%$, que se puede observar en las figuras.

El dolor crónico es hoy día un gran problema de salud y genera un alto índice de incapacidad laboral y que además es un gran costo económico para la población, y la estimulación transcraneal con corriente directa ayudó a los pacientes a disminuir el dolor crónico, sin embargo, ya está siendo investigada en los últimos años. A pesar de que hoy en día es poco conocida y poco utilizada, dio a conocer su gran utilidad y eficacia.

Los resultados enriquecen la comprensión y nos dan un conocimiento respecto a la eficacia de este tratamiento.

\section{Conclusiones}

- Con este artículo se concluye que los pacientes que recibieron estimulación transcraneal con corriente directa en el tratamiento de dolor crónico tuvieron una buena experiencia y mejora en su salud.

- Como demuestra la investigación, el dolor crónico es un importante factor para la incapacidad laboral y de las actividades de la vida diaria, y en cuanto los pacientes iniciaron el tratamiento lograron reintegrarse a sus actividades de la vida diaria sin presentar ninguna dificultad.

- La estimulación transcraneal con corriente directa es una técnica no invasiva, de bajo costo y de gran utilidad para el tratamiento del dolor crónico.

\section{Financiamiento}

La presente investigacion no ha recibido ninguna beca específica de agencias de los sectores públicos, comercial o sin animo de lucro.

\section{Conficto de intereses}

Los autores declaran no tener conflicto de intereses.

\section{Responsabilidades éticas}

Protección de personas y animales. Los autores declaran que para esta investigación no se han realizado experimentos en seres humanos ni en animales.

Confidencialidad de los datos. Los autores declaran que han seguido los protocolos de su centro de trabajo sobre la publicación de datos de pacientes.

Derecho a la privacidad y consentimiento informado. Los autores han obtenido el consentimiento informado de los pacientes y/o sujetos referidos en el artículo. 


\section{G.Y. Medina-Chamu: Estimulación transcraneal}

\section{Bibliografía}

1. IASP terminology [Internet]. International Association for the Study of Pain; 2018. Disponible en: https://www.iasp-pain.org/Education/Content.aspx? ItemNumber $=1698$

2. Bautista Sánchez SG, Jímenez Santiago A. Epidemiología del dolor crónico. Arch Med Fam. 2014;16(4):69-72.

3. Bonica JJ. The management of pain. Filadelfia: Lea \& Febiger; 1953.

4. Treede RD. Entstehung der Schmerzchronifizierung. En: Baron R, Koppert W, Strumpf M, Willweber-Strumpf A, editores. Praktische Schmerztherapie. Heidelberg: Springer; 2011. pp. 3-13.

5. Merskey H, Bogduk N. Classification of chronic pain. 2nd ed. Seattle: IASP Press; 1994. p. 1.

6. Mäntyselkä P, Kumpusalo E, Ahonen R, Kumpusalo A, Kauhanen J Viinamäki $\mathrm{H}$, et al. Pain as a reason to visit the doctor: a study in Finnish primary health care. Pain. 2001;89(2-3):175-80.

7. Goldberg DS, McGee SJ. Pain as a global public health priority. BMC Public Health. 2011;11:770.

8. ecker N, Thomsen AB, Olsen AK, Sjøgren P, Bech P, Eriksen J. Pain epideliology and health related quality of life in chronic non-malignant pain patients referred to a Danish multidisciplinary pain center. Pain. 1997;79:393-400.

9. McDermott AM, Toelle TR, Rowbotham DJ, Schaefer CP, Dukes EM The burden of neuropathic pain: results from a cross-sectional survey. Eur J Pain. 2006;10:127-35.

10 Dunn KM, Croft PR. Epidemiology and natural history of low back pain. Eur Medicophys. 2004:40:9-13.

11. Baron R. Mechanisms of disease: neuropathic pain-a clinical perspective. Nat Clin Pract Neurol. 2006;2(2):95-106.
12. Priori A. Brain polarization in humans: a reappraisal of an old tool for prolonged noninvasive modulation of brain excitability. Clin Neurophysiol. 2003;114:589-95.

13. Nitsche MA, Paulus W. Sustained excitability elevations induced by transcranial DC motor cortex stimulation in humans. Neurology. 2001;57:1899-901.

14. Chhatbar PY, Kautz SA, Takacs I, Rowland NC, Revuelta GJ, George MS, et al. Evidence of transcranial direct current stimulation generated electric fields at sub thalamic level in human brain in vivo. Brain Stimul. 2018;11(4):727-33.

15. Lefaucheur JP, Antal A, Ayache SS, Benninger DH, Brunelin J, Cogiamanian $\mathrm{F}$, et al. Evidence-based guidelines on the therapeutic use of transcranial direct current stimulation (tDCS). Clin Neurophysiol. 2017;128(1):56-92.

16. Lüdemann-Podubecká J, Bösl K, Rothhardt S, Verheyden G, Nowak DA. Transcranial direct current stimulation for motor recovery of upper limb function after stroke. Neurosci Biobehav Rev. 2014;47:245-59.

17. Neuling T, Rach S, Wagner S, Wolters $\mathrm{CH}$, Herrmann CS. Good vibrations: oscillatory phase shapes perception. Neuroimage. 2012;63:771-8.

18. Strüber D, Rach S, Trautmann-Lengsfeld SA, Engel AK, Herrmann CS. Antiphasic $40 \mathrm{~Hz}$ oscillatory current stimulation affects bistable motion perception. Brain Topogr. 2014;27:158-71.

19. Marshall L, Helgadóttir $H$, Mölle $M$, Born J. Boosting slow oscillations during sleep potentiates memory. Nature. 2006;444(7119):610-3.

20. Antonenko D, Diekelmann S, Olsen C, Born J, Mölle M. Napping to renew learning capacity: enhanced encoding after stimulation of sleep slow oscillations. Eur J Neurosci. 2013;37:1142-51.

21. Massetti T, Crocetta TB, Silva TDD, Trevizan IL, Arab C, Caromano FA, et al. Application and outcomes of therapy combining transcranial direct current stimulation and virtual reality: a systematic review. Disabil Rehabil Assist Technol. 2017;12(6):551-9. 